Published in final edited form as:

J Phys Chem A. 2005 May 26; 109(20): 4431-4436.

\title{
Singlet excited-state dynamics of 5-fluorocytosine and cytosine: An experimental and computational study
}

\author{
Lluís Blancafort ${ }^{\star}, \dagger$, Boiko Cohen $\ddagger$, Patrick M. Hare $\ddagger$, Bern Kohler ${ }^{\star}, \ddagger$, and Michael A. Robb§ \\ Institut de Química Computacional, Universitat de Girona, 17071 Girona, Spain, Department of \\ Chemistry, The Ohio State University, 100 W. 18th Avenue, Columbus, OH 43210, and Department \\ of Chemistry, Imperial College London, London, SW7 2AZ, United Kingdom
}

\section{Abstract}

The photophysics of singlet excited 5-fluorocytosine (5FC) was studied in steady-state and timeresolved experiments and theoretically by quantum chemical calculations. Femtosecond transient absorption measurements show that replacement of the $\mathrm{C} 5$ hydrogen of cytosine by fluorine increases the excited-state lifetime by two orders of magnitude from $720 \mathrm{fs}$ to $73 \pm 4 \mathrm{ps}$. Experimental evidence indicates that emission in both compounds originates from a single tautomeric form. The lifetime of $5 \mathrm{FC}$ is the same within experimental uncertainty in the solvents ethanol and dimethylsulfoxide. The insensitivity of the $S_{1}$ lifetime to the protic nature of the solvent suggests that proton transfer is not the principal quenching mechanism for the excited state. Excited state calculations were carried out for the amino-keto tautomer of 5FC, the dominant species in polar environments, in order to understand its longer excited-state lifetime. CASSCF and CAS-PT2 calculations of the excited states show that the minimum energy path connecting the minimum of the ${ }^{1} \pi, \pi^{*}$ state with the conical intersection responsible for internal conversion has essentially the same energetics for cytosine and $5 \mathrm{FC}$, suggesting that both bases decay nonradiatively by the same mechanism. The dramatic difference in lifetimes may be due to subtle changes along the decay coordinate. A possible reason may be differences in the intramolecular vibrational redistribution rate from the Franck-Condon active, in-plane modes to the out-of-plane modes that must be activated to reach the conical intersection region.

\section{Introduction}

DNA damage by solar UV light is responsible for a variety of adverse health effects, including immune suppression, photoaging, and skin cancer. ${ }^{1,2}$ This serious public health problem has been a principal motivation for understanding excited electronic states in nucleic acids. In recent years, considerable progress has been made in characterizing the initial relaxation pathways of single electronically excited bases. ${ }^{3}$ Transient absorption ${ }^{4-6}$ and fluorescence upconversion experiments ${ }^{7-9}$ using femtosecond laser pulses have revealed that the lifetimes of the lowest excited singlet states $\left(\mathrm{S}_{1}\right.$ states) of the naturally occurring bases are subpicosecond in aqueous solution at room temperature.

A growing number of theoretical studies have now been published that address the mechanism behind ultrafast nonradiative decay in the nucleobases. ${ }^{10-18}$ The excited electronic singlet states of cytosine have been the subject of several computational studies. ${ }^{10,14,16-18}$ Based

\footnotetext{
*Corresponding Authors. E-mail:kohler@chemistry.ohio-state.edu,lluisb@stark.udg.es.

†Universitat de Girona

\$The Ohio State University

§Imperial College London
}

Supporting Information Available. List of active spaces used in the calculations, CAS-PT2 energy profiles along the calculated MEP for radiationless decay $\left(\left(\pi, \pi^{*}\right)\right.$ Min to $\left.\left(\mathrm{S}_{1} / \mathrm{S}_{0}\right)_{\mathrm{X}}\right)$, and Cartesian coordinates of important $5 \mathrm{FC}$ structures. 
on CASSCF calculations, Ismail et al. ${ }^{14}$ proposed that nonradiative decay occurs through a conical intersection accessed after a state switch from the optically prepared ${ }^{1} \pi, \pi^{*}$ to a closelying ${ }^{1} \mathrm{n}, \pi^{*}$ state. Merchán and Serrano-Andrés ${ }^{17}$ later questioned the intermediacy of the ${ }^{1} \mathrm{n}$, $\pi^{*}$ state based on their CAS-PT2//CASSCF study. However, a recent re-examination of the problem at the CAS-PT2//CASSCF level of theory suggests that the path that leads to the conical intersection with the ground state involves interaction between three excited states: the ground state, a ${ }^{1} \pi, \pi^{*}$ state, and one ${ }^{1} \mathrm{n}, \pi^{*}$ state. ${ }^{18}$ These calculations give an upper bound of approximately $7 \mathrm{kcal} \mathrm{mol}^{-1}$ to the barrier to access the intersection. Ultrafast nonradiative decay is believed to occur despite this barrier because the barrier top is approximately $12 \mathrm{kcal}$ $\mathrm{mol}^{-1}$ below the Franck-Condon point on the initial excited-state surface. Given the importance of the ${ }^{1} \pi, \pi^{*}$ and ${ }^{1} \mathrm{n}, \pi^{*}$ interaction for the proposed radiationless decay mechanism, any differential shift in the energies of these states could lead to a reduction in their coupling and/ or perturb the barrier that limits access to the conical intersection. Such a shift could be accomplished through covalent modification or a change in solvent.

Here we report an in-depth experimental and computational study of nonradiative decay by the singlet excited state of 5-fluorocytosine (5FC). Previously, it was shown that several cytosine derivatives have dramatically lower rates of nonradiative decay than the natural base. $65 \mathrm{FC}$ was chosen for this study because it differs from cytosine by a single atom substitution, yet has an $\mathrm{S}_{1}$ lifetime that is longer by two orders of magnitude. $5 \mathrm{FC}$ was modeled computationally using the same methods previously applied to cytosine. ${ }^{14,18}$ This allows us to address whether the increased lifetime is the result of readily rationalized changes to the excited state potential energy landscape, or whether it results from a fundamentally different radiationless decay mechanism.

Apart from the desire to understand nonradiative decay in the nucleobases, there are practical motivations for studying the interaction of $5 \mathrm{FC}$ with light. $5 \mathrm{FC}$ has been used for many years as an antifungal drug in humans. Even though photosensitivity is a serious side effect of 5FC therapy, ${ }^{19}$ little is known about the photophysical and photochemical properties of 5FC. More recently, $5 \mathrm{FC}$ has been widely investigated as a promising prodrug in gene therapy. ${ }^{20,21}$ The prodrug 5FC inhibits DNA and RNA synthesis via its intracytoplasmic conversion to 5-

fluorouracil (5FU). Because $5 \mathrm{FU}$ is a major photodegradation product of $5 \mathrm{FC}^{22}$ there is a need to better characterize its excited state properties.

\section{Experimental Methods}

Transient absorption signals were recorded by the pump-probe technique using an amplified titanium-sapphire laser system, as described previously.., 23 Briefly, pump pulses with a center wavelength of $\sim 263 \mathrm{~nm}$ were obtained from the third harmonic of the laser output. Probe pulses were derived from a white light continuum generated in a $1 \mathrm{~cm}$ path length cell filled with water. The difference-frequency mixing signals between the third harmonic pump pulse and the fundamental in the probe arm measured at the sample position had a FWHM of $270 \mathrm{fs}$. From this measurement, the FWHM of the instrument response function was determined to be $\sim 190 \mathrm{fs}$, assuming a Gaussian pulse shape.

Steady-state absorption spectra were recorded using a UV/vis spectrophotometer (Lambda 25, Perkin-Elmer Corp.). The steady-state fluorescence measurements were performed with a commercial fluorimeter (Fluoromax-3, Jobin Yvon, Inc.). Solutions for the transient absorption experiments had an absorbance of approximately 1.0 in a $1 \mathrm{~mm}$ path length at the excitation wavelength of $263 \mathrm{~nm}$. Samples for fluorescence measurements were held in $1 \mathrm{~cm}$ path length cuvettes and had optical densities of less than 0.3 at the excitation wavelength. Fluorescence quantum yields were estimated by the comparison method using either rhodamine B in ethanol 
$\left(\varphi_{\mathrm{f}}=0.65^{24}\right)$ or 2-naphthol at $\mathrm{pH} 10\left(\varphi_{\mathrm{f}}=0.21^{25,26}\right)$ as standards. The slit widths were $2 \mathrm{~nm}$ for both excitation and emission monochromators.

All compounds were purchased from Sigma Chemical Company (St. Louis, MO) and used as received. Solutions were prepared with water from a Barnstead International (Dubuque, IA) model D4741 water purification system. Samples were circulated through a fused silica flow cell with a $1 \mathrm{~mm}$ path length, using a laboratory gear pump. Solute concentrations were adjusted to produce an absorbance between 1.0 (nonaqueous solutions) and 1.5 (aqueous solutions) in a $1 \mathrm{~mm}$ path length at the excitation wavelength of $263 \mathrm{~nm}$.

Measurements at neutral $\mathrm{pH}$ conditions $(\mathrm{pH}=6.8)$ were carried out in buffered solutions, prepared with $0.025 \mathrm{M}$ potassium dihydrogen phosphate and $0.025 \mathrm{M}$ disodium hydrogen phosphate. ${ }^{23}$ Acidic solutions were prepared by dropwise addition of concentrated phosphoric acid. Alkaline solutions were prepared by addition of a small quantity of solid $\mathrm{NaOH}$. Although these latter solutions were unbuffered, $\mathrm{pH}$ measurements carried out before and after the laser experiments showed no change in $\mathrm{pH}$. UV/vis spectra recorded before and after laser data collection showed no evidence of sample degradation.

\section{Computational Details}

The CASSCF/6-31G* calculations were carried out with the Gaussian03 program. ${ }^{27}$ The CASPT2/6-31G* calculations were performed with the MOLCAS program (version 5.2). ${ }^{28}$ The active spaces and number of states considered in the calculations are listed in the Supporting Information. A detailed discussion of the choice of active space and the calculation of reaction paths at the CASSCF level appears in the Supporting Information of our previous theoretical cytosine study. ${ }^{14}$ Briefly, our study here of singlet excited 5FC consists of optimization of critical points (including the ground-state minimum) and calculation of excited-state minimum-energy paths (MEP) with the IRC method, at the CASSCF level. In addition, the relevant $\left(\mathrm{S}_{1} / \mathrm{S}_{0}\right)$ conical intersection is relocated with a linear coordinate calculation at the CAS-PT2 level, and the energetics along the MEP are recalculated at the same level of theory. The same procedure was followed in our recent re-examination of singlet excited cytosine, 18 and the details are described there. The barrier for the alternative decay through the conical intersection between the $\left(\mathrm{n}_{\mathrm{N}}, \pi^{*}\right)$ and the ground state $\left(\left(\mathrm{n}_{\mathrm{N}}, \pi^{*}\right)_{\text {Barr }}\right)$, at the CAS-PT2 level, was determined by calculating the central section of the MEP at the $\operatorname{CASSCF}(8,7) / 6-31 \mathrm{G}^{*}$ level and recomputing the CAS-PT2 energetics. The CAS-PT2 barrier for the photochemically induced dissociation of the fluorine-carbon bond $\left((\mathrm{C}-\mathrm{F})_{\mathrm{Barr}}\right)$ was determined analogously, calculating the MEP at the $\operatorname{CASSCF}(8,8) / 6-31 \mathrm{G}^{*}$ level.

\section{Results}

\subsection{Steady-state measurements}

Figure 1 compares the absorption spectra of cytosine and 5FC in aqueous solution at $\mathrm{pH} 6.8$. The absorption maximum of the neutral form of $5 \mathrm{FC}$ occurs at $36400 \mathrm{~cm}^{-1}(275 \mathrm{~nm})$ and is significantly red shifted compared to cytosine, which has maximum absorption at 37600 $\mathrm{cm}^{-1}(266 \mathrm{~nm})$. Figure 1 also shows the corresponding emission spectra recorded at an excitation wavelength of $263 \mathrm{~nm}$. The emission spectra show no vibrational fine structure in keeping with results for other DNA bases in aqueous solution. ${ }^{29}$ Emission from 5FC in ethanol was also recorded, but differs insignificantly from that in water (data not shown). The fluorescence quantum yield of 5FC in aqueous solution at $\mathrm{pH} 6.8$ was determined to be 0.005 (10\% estimated random error). Furthermore, the fluorescence quantum yield was found to be independent of excitation wavelength over the range studied $(265-290 \mathrm{~nm})$. A significantly larger fluorescence Stokes shift of $8600 \mathrm{~cm}^{-1}$ is observed for 5FC compared to the value of 
$6500 \mathrm{~cm}^{-1}$ seen for cytosine. This could indicate a large geometry change in the $S_{1}$ state or a change in electronic character. These possibilities will be addressed in the discussion section.

\subsection{Transient Absorption of 5FC}

Figure 2 compares transient absorption signals for cytosine and 5FC pumped at $263 \mathrm{~nm}$ and probed at $570 \mathrm{~nm}$. It is clear from this figure that replacing the $\mathrm{C} 5$ hydrogen of cytosine by fluorine dramatically increases the excited state lifetime, as reported previously. 6 Transient absorption signals were recorded at three separate probe wavelengths in $\mathrm{pH} 6.8$ aqueous solution and corrected for two-photon ionization of water, as described elsewhere. ${ }^{23} \mathrm{~A}$ global fit to the corrected transients gave a lifetime of $73 \pm 4$ ps (Figure 3).

The radiative lifetime, $\tau_{\text {rad }}$, for $5 \mathrm{FC}$ was calculated from the steady-state absorption and emission spectra using the Strickler-Berg equation. ${ }^{30}$ First, a Gaussian was fit to the lowest energy absorption band plotted vs. wavenumbers and used to evaluate the integral over the extinction coefficient in the Strickler-Berg equation. Next, the required integral over the emission spectrum was approximated by the cube of the wavenumber of maximum emission, as in our earlier report. ${ }^{31}$ The radiative lifetime estimated in this way is $14 \mathrm{~ns}$.

Pump-probe measurements on 5FC were also carried out in two nonaqueous solvents. Figure 4 compares the transient absorption signals at $570 \mathrm{~nm}$ in ethanol, DMSO and water. As shown in this figure, the same lifetime was observed in all solvents within experimental uncertainty. In agreement with this finding, the fluorescence quantum yield was observed to be independent of solvent.

\subsection{Excited-State Calculations}

To gain insight into the two-order-of-magnitude difference between the $\mathrm{S}_{1}$ lifetimes of 5FC and its parent compound, the excited-state potential energy surface of the fluorinated derivative was computed at the CASSCF level of theory, and the energetics recalculated at the CAS-PT2 level. The recalculated CAS-PT2 energy profile along the decay MEP is shown schematically in Figure 5. The figure shows the relative energies at the relevant critical points for $5 \mathrm{FC}$, with the corresponding values for cytosine in parentheses (see also Table 2). The three main parameters that should dominate the decay dynamics, namely the excess vibrational energy (energy difference between the Franck-Condon point and the minimum of the $\pi, \pi^{*}$ surface), the barrier to access the intersection from $\left(\pi, \pi^{*}\right)_{\text {Min }}$ and the relative energy of $\left(\mathrm{S}_{1} / \mathrm{S}_{0}\right)_{\mathrm{X}}$ are very similar for the two compounds, with relative differences of less than $2 \mathrm{kcal} \mathrm{mol}^{-1}$. In addition, the optimized structures for 5FC have bond lengths and angles in the ring which are very similar to the ones in cytosine and are in agreement with the valence-bond structures discussed previously. ${ }^{14,18}$ As discussed in previous theoretical studies on cytosine, ${ }^{14,18}$ the nuclear coordinate consists of bond inversion in the ring and pyramidalization of the unsubstituted C6 carbon, and along the final part of the decay the states have a mixed ${ }^{1} \pi, \pi^{*}$ and ${ }^{1} \mathrm{n}, \pi^{*}$ character due to the proximity effect. We conclude that differences in the decay mechanism are unlikely to explain the different fluorescence lifetimes for the two compounds. Indeed, the similar potential energy surfaces revealed by the calculations suggest that they follow a common decay path.

In addition to the mixed $\left(\pi, \pi^{*} / \mathrm{n}_{\mathrm{O}}, \pi^{*}\right)$ path, additional decay paths via the $\left(\mathrm{n}_{\mathrm{N}}, \pi^{*}\right)$ state 14 and via fluorine-carbon dissociation were calculated (see Table 2). The barrier for the alternative decay through the conical intersection between the $\left(\mathrm{n}_{\mathrm{N}}, \pi^{*}\right)$ and the ground state is higher than the one for the first path ( $13.6 \mathrm{kcal} \mathrm{mol}^{-1}$ for $5 \mathrm{FC}$ and $7.8 \mathrm{kcal} \mathrm{mol}^{-1}$ for cytosine). The estimated CAS-PT2 barrier for the photochemically induced fluorine-carbon dissociation is $35.8 \mathrm{kcal} \mathrm{mol}^{-1}$. From the last result we conclude that photolysis of 5FC is energetically feasible only for excitation energies at least $1 \mathrm{eV}$ higher than the vertical transition energy. 


\section{Discussion}

No value for the extinction coefficient of neutral $5 \mathrm{FC}$ was found in the literature, prompting our measurement. Our value of $5900 \mathrm{M}^{-1} \mathrm{~cm}^{-1}$ at $\lambda_{\max }=275 \mathrm{~nm}$ indicates that 5-fluorination slightly decreases the intensity of the long wavelength absorption band compared to cytosine $\left(\varepsilon=6100 \mathrm{M}^{-1} \mathrm{~cm}^{-1}\right.$ at $\left.\lambda_{\max }=266 \mathrm{~nm}^{32}\right)$. The same trend is seen for the nucleoside of 5FC: The extinction coefficient for 5-fluorocytidine is $8060 \mathrm{M}^{-1} \mathrm{~cm}^{-1}$ at $\lambda_{\max }=281 \mathrm{~nm}^{33}$ compared to $9100 \mathrm{M}^{-1} \mathrm{~cm}^{-1}$ at $\lambda_{\max }=271 \mathrm{~nm}$ for cytidine. ${ }^{32}$

Substitution of hydrogen by fluorine significantly increases the $\mathrm{S}_{1}$ lifetime of cytosine at $\mathrm{pH}$ 6.8 from $720 \mathrm{fs}^{5}$ to $73 \pm 4 \mathrm{ps}$. Malone et al. ${ }^{6}$ reported a lifetime of $88 \pm 5 \mathrm{ps}$. In that study, the two-photon ionization of water was modeled by a constant offset and was not subtracted by the more rigorous procedure used in this and our other recent works. ${ }^{23,34}$ The value reported here is more accurate in our opinion because the new subtraction procedure correctly accounts for the dynamics of the water ionization signal, which has decay components similar in magnitude to the $\mathrm{S}_{1}$ lifetime.

The fluorescence quantum yield of $5 \mathrm{FC}$ is $\approx 60$ times greater than the quantum yield reported for cytosine by Daniels and Hauswirth ${ }^{35}\left(\varphi_{\mathrm{f}}=8 \times 10^{-5}\right)$. Although fluorescence from 5FC has not been reported previously to the best of our knowledge, fluorescence from the $5 \mathrm{FC}$ anion generated in alkaline solution has been used as an assay for $5 \mathrm{FC}$ concentrations in blood. ${ }^{36}$, 37 The increased quantum yield of 5FC indicates that the more slowly decaying signals seen in the transient absorption measurements are indeed attributable to the fluorescent excited state. This is also supported by the calculated radiative lifetime of $14 \mathrm{~ns}$. Combining this value with the measured fluorescence quantum yield of 0.005 gives a predicted fluorescence lifetime of $70 \mathrm{ps}$, in excellent agreement with the experimental value. It was shown previously that the experimentally measured $S_{1}$ lifetimes of the natural bases generally agree very well with ones calculated from the Strickler-Berg equation. ${ }^{31}$ This good agreement provides strong evidence that the emission from $5 \mathrm{FC}$ is entirely from the ${ }^{1} \pi, \pi^{*}$ state prepared by optical excitation. It thus rules out other potential excited-state quenching mechanisms such as prompt carbonfluorine photodissociation. As discussed above, calculations show that carbon-fluorine scission is unlikely on energetic grounds.

Calculations were performed exclusively on the amino-keto tautomers of cytosine and 5FC (structures shown in Figure 1). There is consensus in the literature that these are the lowest energy tautomers in a polar environment. ${ }^{38,39}$ Occasionally, emission from a nucleobase is dominated by fluorescence from a minor tautomer. For example, $7 \mathrm{H}$-adenine, although present in low concentration in an aqueous solution of adenine, is responsible for nearly all of the emission. ${ }^{23,40}$ We considered whether a minor tautomer, which may be much more abundant for $5 \mathrm{FC}$ than for cytosine, could account for the increased lifetime of the former compound.

Several considerations allow us to rule out fluorescence from a minor tautomer in the case of 5FC. First, good agreement of the experimentally measured fluorescence lifetime with the one calculated from Strickler-Berg analysis is consistent with emission by nearly $100 \%$ of the excited molecules. Second, our previous work with adenine has shown that decays corresponding to individual tautomers with different fluorescence lifetimes are easily resolved in femtosecond transient absorption experiments. ${ }^{23}$ A less fluorescent tautomer would have been observable as a fast decay component, yet all of our signals can be fit by a single exponential, consistent with a single tautomer. Third, moderate changes in solvent polarity should have a relatively small effect on the concentration and properties of the major tautomer, but a large effect on minor ones. In the present experiments, the fluorescence lifetime and quantum yield do not vary with solvent polarity as seen in Figure 4. Finally, emission by 5FC is independent of excitation wavelength. Because the absorption spectra of different tautomers 
are slightly shifted with respect to each other, varying the excitation wavelength leads to changes in the fluorescence quantum yield, as seen for adenine. ${ }^{40}$ The photophysical properties of 5FC thus reflect the excited-state dynamics of a single predominant species, the amino-keto tautomer.

The CAS-PT2 calculations predict only a small decrease of $0.06 \mathrm{eV}$ for the vertical excitation energy of 5FC compared to cytosine. Experimentally, a somewhat larger decrease is observed. There are no other published calculations on 5FC to compare these results with, however the vertical singlet-triplet energy gap of uracil was observed to decrease by $0.2 \mathrm{eV}$ in DFT calculations comparing 5FU with uracil. ${ }^{41}$ The absolute transition energies computed for $5 \mathrm{FC}$ and cytosine using the CAS-PT2 method compare favorably with experiment (see Table 1).

The Stokes shift was calculated as the difference between the vertical transition energy for absorption and the vertical transition energy for (relaxed) emission. The latter quantity is just the energy difference between the $S_{1}$ and $S_{0}$ surfaces, evaluated at the geometry of $\left(\pi, \pi^{*}\right)_{\text {Min }}$. CAS-PT2 calculations gave $2.52 \mathrm{eV}$ for the vertical emission energy of cytosine and $2.38 \mathrm{eV}$ for 5FC. The calculated Stokes shift for 5FC is larger than for cytosine, consistent with the experimental trend, but the absolute values are twice as large (Table 3). The poor agreement can be rationalized by the flatness of the $S_{1}$ surface in a region of nuclear coordinate space where the $S_{0}$ surface is rising sharply in energy. Thus, uncertainties in precisely locating the

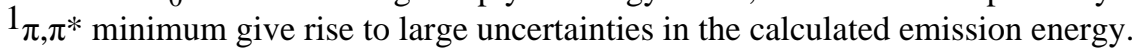

We now turn our attention to the primary objective of this work, which is to ascertain the reasons for the much longer lifetime of 5FC. The question arises whether a change in the nonradiative decay mechanism takes place upon substitution at C5. If proton transfer in the excited state were the rate-limiting step, then fluorination, which frequently leads to profound changes in acid/base properties, could profoundly influence the decay dynamics. For this reason, 5FC was studied in ethanol and dimethylsulfoxide (DMSO) and the results were compared with those in water. Ethanol is a hydrogen-bonding solvent of lower polarity than water, while DMSO is a weakly basic, polar aprotic solvent. The transient absorption signals were the same in all three solvents within experimental uncertainty, suggesting that there is no significant solvent coordinate in the decay. This behavior contrasts with the pronounced solvent effects frequently observed for intermolecular excited-state proton transfer. ${ }^{42}$ Because of the lack of experimental evidence for excited-state proton transfer, this decay pathway was not studied computationally. Experimental evidence inconsistent with excited-state proton transfer was previously reported for adenine. ${ }^{23}$ Finally, the fact that similar dynamics are observed in aqueous and nonaqueous solvents is consistent with the sole presence of the neutral form of 5FC. 43

On the basis of the present CASSCF/CAS-PT2 calculations, cytosine and its 5-fluorinated derivative appear to decay by a common mechanism that requires passage over a low barrier to reach a conical intersection with the ground state. In principle, the increase of fluorescence lifetime in 5FC could be due to a larger barrier height along the decay path, or to a smaller excess vibrational energy, compared to cytosine. However, the calculated values for the two compounds are approximately the same, within the accuracy of the calculations. Therefore the key to understanding the very different rates of nonradiative decay, could be the flow of energy from the Franck-Condon active modes to the modes responsible for barrier crossing. In the present case, this would mean intramolecular vibrational energy redistribution (IVR) from the in-plane modes corresponding to bond-inversion, which are activated along the decay coordinate to $\left(\pi, \pi^{*}\right)_{\text {Min }}$, to out-of-plane modes, corresponding to pyramidalization of C6. According to this hypothesis, the rate at which energy is reorganized from the Franck-Condon active modes into the coordinates responsible for motion toward the conical intersection would be much faster for cytosine than for 5FC. In fact, Leitner and Wolynes have emphasized the 
importance of dynamical corrections to isomerization reactions, particularly those with low barriers. ${ }^{44}$ However, it is unclear how the presence of a fluorine atom on a carbon atom adjacent to the one undergoing pyramidalization can affect IVR so drastically as to change the fluorescence lifetime by two orders of magnitude. Dynamical simulations of energy flow due to IVR and vibrational energy transfer to the solvent could provide further insight.

Alternative explanations for the different fluorescence lifetimes are possible. One such explanation could be inaccuracies in the calculated potential energy surfaces. Here the calculated barriers are only upper bounds to the actual values, since they were determined using CAS-PT2 single-point calculations and were not optimized at that level. The close agreement between the calculated barriers along the decay path for the two compounds could therefore be accidental. Because of these inaccuracies, we cannot exclude that the barrier for 5FC is actually higher than the one for cytosine, contributing to its longer fluorescence lifetime. Solvation effects, which were not included in the present calculations, is a further possibility. On the other hand, the effect of the alternative path via the $\left(\mathrm{n}_{\mathrm{N}}, \pi^{*}\right)$ state in the different photophysics appears to be small. For cytosine, the mixed $\left(\pi, \pi^{*} / \mathrm{n}_{\mathrm{O}} \pi^{*}\right)$ path is preferred by $1.9 \mathrm{kcal} \mathrm{mol}^{-1}$ (see Table 2), while the difference for $5 \mathrm{FC}$ is higher $\left(7.7 \mathrm{kcal} \mathrm{mol}^{-1}\right)$. Thus a fraction of excited cytosine molecules may decay along the $\left(\mathrm{n}_{\mathrm{N}}, \pi^{*}\right)$ path, but this path should be virtually suppressed for 5FC. However, since only a small fraction of cytosine molecules will follow this decay route, the $\left(\mathrm{n}_{\mathrm{N}}, \pi^{*}\right)$ path can account at best for a small part of the total decay.

\section{Conclusions}

Substitution of hydrogen at C5 by fluorine increases the singlet excited state lifetime by a factor of 100. In aqueous solution, the emission from 5FC is independent of excitation wavelength between 265 and $290 \mathrm{~nm}$, and the measured fluorescence lifetime is in good agreement with the value predicted from Strickler-Berg analysis. These observations are consistent with the presence of a single tautomer, which emits from its lowest energy ${ }^{1} \pi, \pi^{*}$ state. Past work 38 , 39 indicates that this tautomer is the amino-keto form. Calculations suggest that the aminoketo tautomers of 5FC and cytosine decay by the same radiationless decay mechanism. However, the energetics along the decay path are very similar, and the calculations cannot explain the different fluorescence lifetimes. A possible reason may be that the present level of theory is not accurate enough to reproduce eventual differences in the energies along the path. A further hypothesis is that the two molecules have different rates of energy flow into the outof-plane modes that must be excited to access the conical intersection responsible for the decay. However, dynamical calculations necessary to assess this possibility are beyond the scope of this paper. Finally, the greater fluorescence of 5FC and the possibility of exciting this modified base at longer wavelengths may offer novel possibilities for studying electronic energy relaxation in base multimers.

\section{Singlet excited-state dynamics of 5-fluorocytosine and cytosine: An experimental and computational study}

Lluís Blancafort, Boiko Cohen, Patrick M. Hare, Bern Kohler,and Michael A. Robb

\section{SUPPORTING INFORMATION}

Supporting Information Available: List of active spaces used in the calculations, details of IRC calculations and Cartesian Coordinates of new cytosine and 5-fluorocytosine structures (PDF). 


\section{Supplementary Material}

Refer to Web version on PubMed Central for supplementary material.

\section{Acknowledgements}

The authors thank Prof. Dongping Zhong (Ohio State University) for the use of his fluorimeter. L. B. is financed by the Ramón y Cajal program from the Spanish Ministerio de Ciencia y Tecnología and by Grants No. BQU2002-04112C02-02 and BQU2002-03334 from the Dirección General de Enseñanza Superior e Investigación Científica y Técnica (MEC-Spain). BC, PMH, and BK are grateful for support by the National Institutes of Health (R01 GM64563).

\section{References}

1. Taylor JS. J Chem Ed 1990;67:835.

2. Kraemer KH. Proc Natl Acad Sci USA 1997;94:11. [PubMed: 8990152]

3. Crespo-Hernández CE, Cohen B, Hare PM, Kohler B. Chem Rev 2004;104:1977. [PubMed: 15080719]

4. Pecourt JML, Peon J, Kohler B. J Am Chem Soc 2000;122:9348.

5. Pecourt JML, Peon J, Kohler B. J Am Chem Soc 2001;123:10370. [PubMed: 11603988]

6. Malone RJ, Miller AM, Kohler B. Photochem Photobiol 2003;77:158. [PubMed: 12785054]

7. Peon J, Zewail AH. Chem Phys Lett 2001;348:255.

8. Gustavsson T, Sharonov A, Markovitsi D. Chem Phys Lett 2002;351:195.

9. Gustavsson T, Sharonov A, Onidas D, Markovitsi D. Chem Phys Lett 2002;356:49.

10. Fülscher MP, Roos BO. J Am Chem Soc 1995;117:2089.

11. Lorentzon J, Fülscher MP, Roos BO. J Am Chem Soc 1995;117:9265.

12. Fülscher MP, Serrano-Andrés L, Roos BO. J Am Chem Soc 1997;119:6168.

13. Broo A, Holmén A. J Phys Chem A 1997;101:3589.

14. Ismail N, Blancafort L, Olivucci M, Kohler B, Robb MA. J Am Chem Soc 2002;124:6818. [PubMed: 12059190]

15. Sobolewski AL, Domcke W, Dedonder-Lardeux C, Jouvet C. Phys Chem Chem Phys 2002;4:1093.

16. Shukla MK, Leszczynski J. J Phys Chem A 2002;106:11338.

17. Merchán M, Serrano-Andrés L. J Am Chem Soc 2003;125:8108. [PubMed: 12837073]

18. Blancafort L, Robb MA. J Phys Chem A 2004;108:10609.

19. Shelley WB, Sica PA Jr. J Am Acad Dermatol 1983;8:229. [PubMed: 6826816]

20. Adachi Y, Tamiya T, Ichikawa T, Terada Ky, Ono Y, Matsumoto K, Furuta T, Hamada H, Ohmoto T. Human Gene Therapy 2000;11:77. [PubMed: 10646641]

21. Nakamura H, Mullen JT, Chandrasekhar S, Pawlik TM, Yoon SS, Tanabe KK. Cancer Res 2001;61:5447. [PubMed: 11454690]

22. Thoma K, Kubler N, Reimann E. Pharmazie 1997;52:455.

23. Cohen B, Hare PM, Kohler B. J Am Chem Soc 2003;125:13594. [PubMed: 14583057]

24. Kubin RF, Fletcher AN. J Lumines 1982;27:455.

25. Lippert E, Nagele W, Seibold-Blankenstein I, Staiger U, Voss W. Z Anal Chem 1959;170:1.

26. Melhuish WH. J Opt Soc Am 1964;54:183.

27. Frisch, M. J.; Trucks, G. W.; Schlegel, H. B.; Scuseria, G. E.; Robb, M. A.; Cheeseman, J. R.; Montgomery Jr., J. A.; Vreven, T.; Kudin, K. N.; Burant, J. C.; Millam, J. M.; Iyengar, S. S.; Tomasi, J.; Barone, V.; Mennucci, B.; Cossi, M.; Scalmani, G.; Rega, N.; Petersson, G. A.; Nakatsuji, H.; Hada, M.; Ehara, M.; Toyota, K.; Fukuda, R.; Hasegawa, J.; Ishida, M.; Nakajima, T.; Honda, Y.; Kitao, O.; Nakai, H.; Klene, M.; Li, X.; Knox, J. E.; Hratchian, H. P.; Cross, J. B.; Adamo, C.; Jaramillo, J.; Gomperts, R.; Stratmann, R. E.; Yazyev, O.; Austin, A. J.; Cammi, R.; Pomelli, C.; Ochterski, J. W.; Ayala, P. Y.; Morokuma, K.; Voth, G. A.; Salvador, P.; Dannenberg, J. J.; Zakrzewski, V. G.; Dapprich, S.; Daniels, A. D.; Strain, M. C.; Farkas, O.; Malick, D. K.; Rabuck, A. D.; Raghavachari, K.; Foresman, J. B.; J. V. Ortiz, J. V.; Cui, Q.; Baboul, A. G.; Clifford, S.; Cioslowski, J.; Stefanov, B. B.; Liu, G.; Liashenko, A.; Piskorz, P.; Komaromi, I.; Martin, R. L.; Fox, D. J.; Keith, T.; Al-Laham, M. A.; Peng, C. Y.; Nanayakkara, A.; Challacombe, M.; Gill, P. M. 
W.; Johnson, B.; Chen, W.; Wong, M. W.; González, C.; Pople, J. A. Gaussian 03 (Revision B.02); Gaussian, Inc.: Pittsburgh, PA, 2003.

28. Andersson, K.; Barysz, M.; Bernhardsson, A.; Blomberg, M. R. A.; Carissan, Y.; Cooper, D. L.; Cossi, M.; Fleig, T.; Fülscher, M. P.; Gaggliarrdi, L.; de Graaf, C.; Hess, B. A.; Karlström, G.; Lindh, R.; Malmquist, P.-Å.; Neogrády, P.; Olsen, J.; Roos, B. O.; Schimmelpfennig, B.; Schütz, M.; Seijo, L.; Serrano-Andrés, L.; Siegbahn, P. E. M.; Stålring, J.; Thornsteinsson, T.; Veryazov, V.;

Wierzbowska, M.; Widmark, P.-O. MOLCAS, VERSION 5.2; Lund University, Sweden, 2001.

29. Parkanyi C, Bouin D, Shieh DC, Tunbrant S, Aaron JJ, Tine A. J Chim Phys Phys-Chim Biol 1984;81:21.

30. Strickler SJ, Berg RA. J Chem Phys 1962;37:814.

31. Cohen B, Crespo-Hernández CE, Kohler B. Faraday Discuss 2004;127:137. [PubMed: 15471343]

32. Voet D, Gratzer WB, Cox RA, Doty P. Biopolymers 1963;1:193.

33. Wempen I, Duschinsky R, Kaplan L, Fox JJ. J Am Chem Soc 1961;83:4755.

34. Crespo-Hernández CE, Kohler B. J Phys Chem B 2004;108:11182.

35. Daniels M, Hauswirth W. Science 1971;171:675. [PubMed: 5540307]

36. Wade D, Sudlow G. J Pharm Sci 1973;62:828. [PubMed: 4705697]

37. Richardson RA. Clin Chim Acta 1975;63:109. [PubMed: 240518]

38. Kulikowski T, Shugar D. Acta Biochimica Polonica 1979;26:145. [PubMed: 506614]

39. Jaworski A, Szczesniak M, Szczepaniak K, Kubulat K, Person WB. J Mol Struct 1990;223:63.

40. Callis PR. Ann Rev Phys Chem 1983;34:329.

41. Nguyen MT, Zhang R, Nam PC, Ceulemans A. J Phys Chem A 2004;108:6554.

42. Borgis D, Hynes JT. J Phys Chem 1996;100:1118.

43. By spectrophotometric titration we measured a $\mathrm{pK}_{\mathrm{a}}$ of 10.2 for neutral $5 \mathrm{FC}$. This value is approximately 2 units lower than for cytosine, but is still too high to produce any significant amount of anions at $\mathrm{pH} 6.8$.

44. Leitner DM, Wolynes PG. Chem Phys Lett 1997;280:411. 

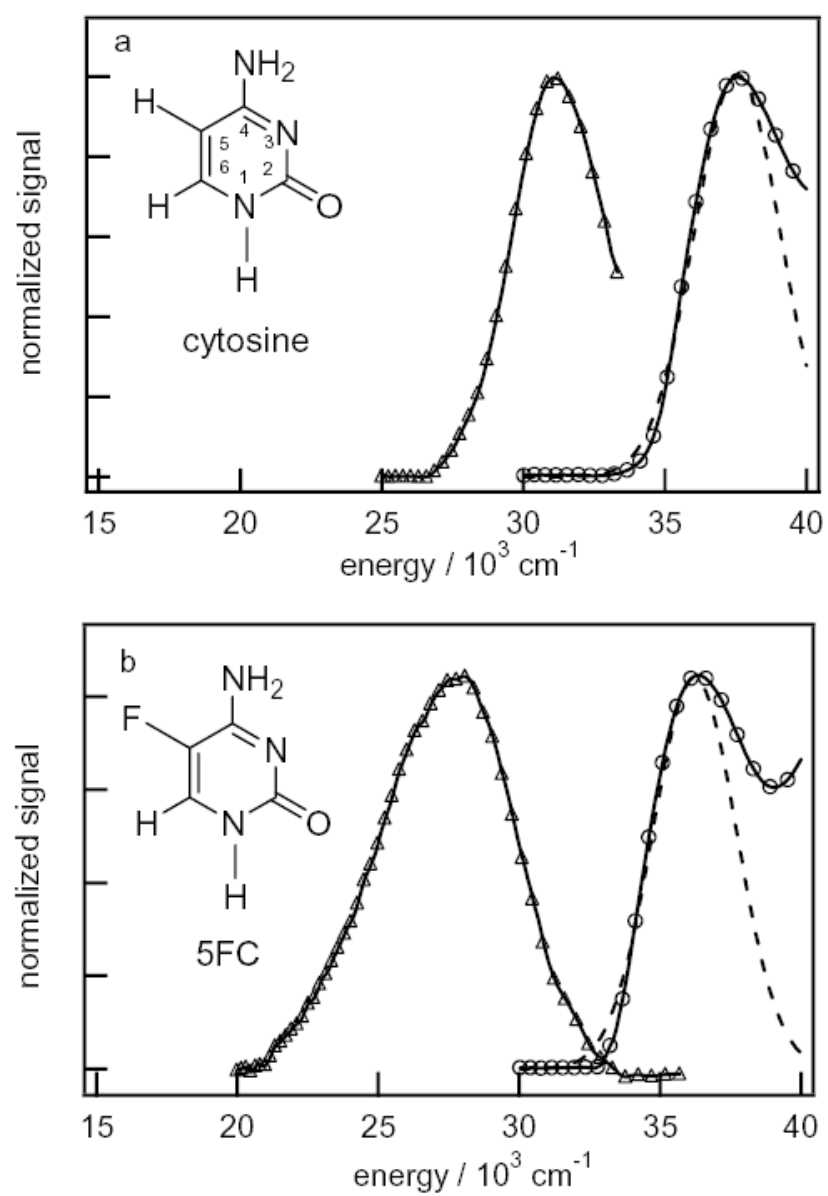

Figure 1.

Normalized absorption (circles) and emission (triangles) spectra of a) cytosine and b) 5FC in water at pH 6.8 together with the Gaussian fits (dashed lines) used for calculating radiative lifetimes by Strickler-Berg analysis. 


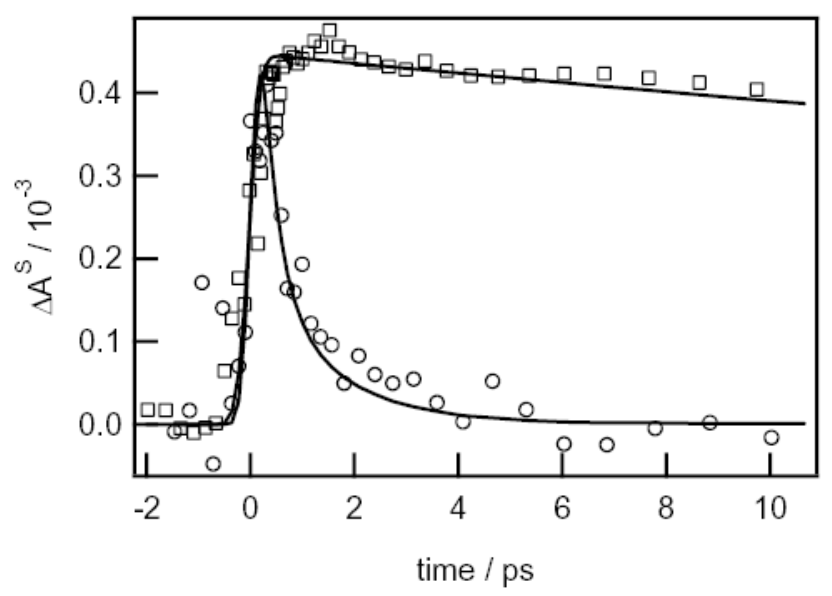

Figure 2.

Comparison of the transient absorption signals of 5FC (squares) and cytosine (circles) excited at $263 \mathrm{~nm}$ and probed at $570 \mathrm{~nm}$. The $5 \mathrm{FC}$ signal was scaled to have the same maximum amplitude as that of cytosine. The signal contribution from two-photon ionization of the solvent has been subtracted from these transients (and in later figures) as indicated by $\Delta \mathrm{As}$. 


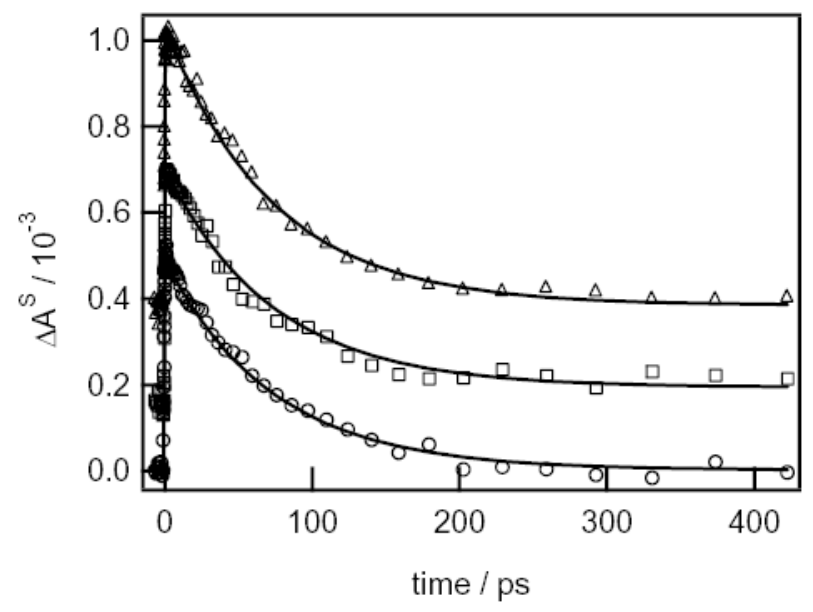

Figure 3.

Transient absorption signals of 5FC excited at $263 \mathrm{~nm}$ and probed at 570 (circles), 600 (squares) and 630 (triangles) nm along with global fits (solid curves). The transients have been vertically offset for clarity. 


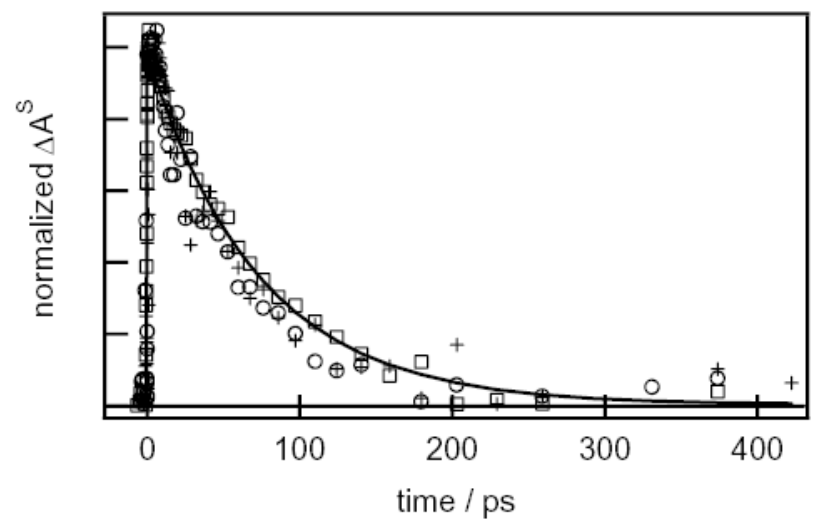

Figure 4.

Transient absorption signals of 5FC pumped at $263 \mathrm{~nm}$ and probed at $570 \mathrm{~nm}$ in DMSO (crosses), EtOH (circles) and water (squares). The solid line is a monoexponential fit. 


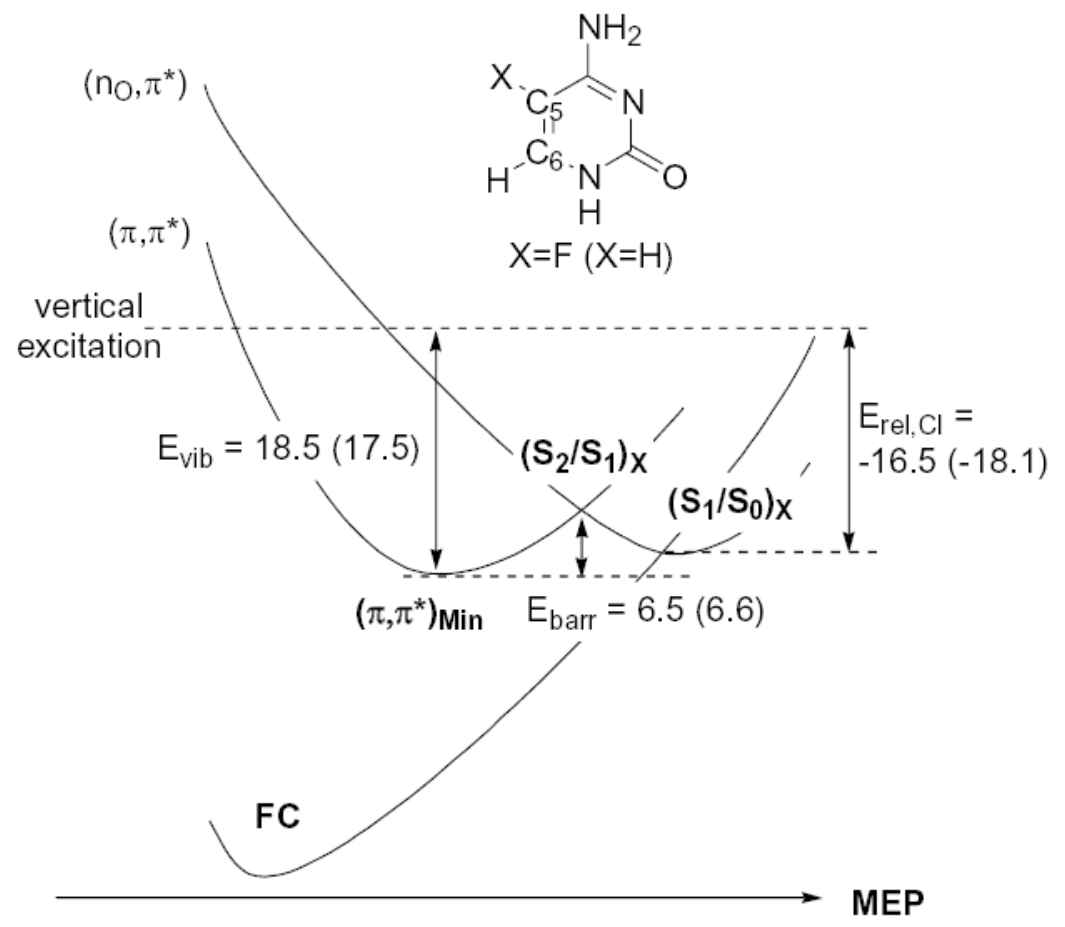

Figure 5.

Energy profiles of the radiationless decay paths (mixed $\left(\pi, \pi^{*} / \mathrm{nO}, \pi^{*}\right)$ path) for $5 \mathrm{FC}$ and cytosine. Energy differences for $5 \mathrm{FC}$ are given in kcal mol-1 with corresponding values for cytosine in parentheses. 
TABLE 1

Vertical excitation energies (eV) of cytosine and 5-fluorocytosine (5FC), calculated at the CASSCF(14,10)/ 6-31G* and CAS-PT2/6-31G* levels of theory.

\begin{tabular}{lcccccc}
\hline State & $\begin{array}{l}\text { cytosine } \\
\text { CASSCF }\end{array}$ & CAS-PT2 & Expt. $^{\boldsymbol{a}}$ & $\begin{array}{l}\text { 5FC } \\
\text { CASSCF }\end{array}$ & CAS-PT2 & Expt. $^{\boldsymbol{a}}$ \\
\hline$\pi, \pi^{*}$ & 5.44 & 4.50 & 4.66 & 5.45 & 4.44 & 4.51 \\
$\mathrm{n}_{\mathrm{O}, \pi^{*}}$ & 5.42 & 4.90 & & 5.40 & 4.88 & \\
$\mathrm{n}_{\mathrm{N}, \pi^{*}}$ & 5.74 & 5.24 & & 5.67 & & \\
\hline
\end{tabular}

$a_{\max }$ in absorption spectrum. Data from this study and ref 32 . 
TABLE 2

Energies $\left(\mathrm{kcal} \mathrm{mol}^{-1}\right)$ of critical points for $5 \mathrm{FC}$ and cytosine (in parentheses). ${ }^{a}$

\begin{tabular}{|c|c|}
\hline & $\mathbf{E}_{\mathrm{rel}}^{b}$ \\
\hline $\begin{array}{l}\left(\pi, \pi^{*}\right) \text { Min } \\
(\mathbf{S} 2 / \mathbf{S} 1) \mathbf{X} \\
(\mathbf{S} 1 / \mathbf{S} 0) \mathbf{X} \\
\left(\mathbf{n N}, \pi^{*}\right) \text { Barr } \\
\left(\mathbf{n N}, \pi^{*}\right) \mathbf{C I} \\
(\mathbf{C}-\mathbf{F}) \text { Barr }\end{array}$ & $\begin{array}{c}-18.5(-17.5) c d \\
-12.0(-10.9) \\
-16.5(-18.1) \\
4.9(-9.7) \\
-14.5(-15.6) \\
16.6(-)\end{array}$ \\
\hline
\end{tabular}

\footnotetext{
${ }^{a}$ Structures optimized at CASSCF/6-31G* level, energies calculated at CAS-PT2/6-31G* level of theory. Energetics for cytosine shown in parentheses.

${ }^{b}$ Energy relative to vertical excitation of $\left(\pi, \pi^{*}\right)$ spectroscopic state.

${ }^{c}$ Corresponds to $3.64 \mathrm{eV} \mathrm{0-0} \mathrm{transition} \mathrm{(band} \mathrm{origin).}$

${ }^{d}$ Corresponds to $3.74 \mathrm{eV} \mathrm{0-0} \mathrm{transition} \mathrm{(band} \mathrm{origin).}$

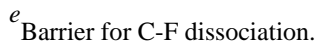


TABLE 3

Photophysical quantities $\left(\mathrm{cm}^{-1}\right)$ in aqueous solution at $\mathrm{pH} 6.8 .^{a}$

\begin{tabular}{|c|c|c|c|c|}
\hline Compound & Abs. max. ${ }^{b}$ & Em. max. ${ }^{c}$ & Experimental Stokes Shift & Calculated Stokes Shift \\
\hline $\begin{array}{l}\text { Cytosine } \\
\text { 5FC }\end{array}$ & $\begin{array}{l}37600(4.66) \\
36400(4.51)\end{array}$ & $\begin{array}{l}31100(3.86) \\
27800(3.45)\end{array}$ & $\begin{array}{l}6500(0.81) \\
8600(1.07)\end{array}$ & $\begin{array}{l}16000(1.98) \\
16600(2.06)\end{array}$ \\
\hline
\end{tabular}

